\title{
Quantitative trait loci analysis reveals candidate genes implicated in regulating functional deficit and CNS vascular permeability in CD8 $\mathrm{T}$ cell-initiated blood-brain barrier disruption
}

\author{
Holly L Johnson ${ }^{1,2,3}$, Lisa M Hanson², Yi Chen", Allan J Bieber ${ }^{1}$, Russell J Buono ${ }^{5}$, Thomas N Ferraro6
} Istvan Pirko ${ }^{1}$ and Aaron J Johnson ${ }^{1,2^{*}}$

\begin{abstract}
Background: Blood-brain barrier (BBB) disruption is an integral feature of numerous neurological disorders. However, there is a relative lack of knowledge regarding the underlying molecular mechanisms of immune-mediated BBB disruption. We have previously shown that CD8 T cells and perforin play critical roles in initiating altered permeability of the BBB in the peptide-induced fatal syndrome (PIFS) model developed by our laboratory. Additionally, despite having indistinguishable CD8 T cell responses, C57BL/6J (B6) mice are highly susceptible to PIFS, exhibiting functional motor deficits, increased astrocyte activation, and severe CNS vascular permeability, while 129S1/SvImJ (129S1) mice remain resistant. Therefore, to investigate the potential role of genetic factors, we performed a comprehensive genetic analysis of (B6 x 129S1) F2 progeny to define quantitative trait loci (QTL) linked to the phenotypic characteristics stated above that mediate CD8 T cell-initiated BBB disruption.

Results: Using single nucleotide polymorphism (SNP) markers and a 95\% confidence interval, we identified one QTL (PIFS1) on chromosome 12 linked to deficits in motor function (SNP markers rs6292954, rs13481303, rs3655057, and rs 13481324, LOD score $=3.3$ ). In addition we identified a second QTL (PIFS2) on chromosome 17 linked to changes in CNS vascular permeability (SNP markers rs6196216 and rs3672065, LOD score $=3.7$ ).

Conclusions: The QTL critical intervals discovered have allowed for compilation of a list of candidate genes implicated in regulating functional deficit and CNS vascular permeability. These genes encode for factors that may be potential targets for therapeutic approaches to treat disorders characterized by CD8 T cell-mediated BBB disruption.
\end{abstract}

Keywords: Quantitative trait loci (QTL), Single nucleotide polymorphism (SNP), Blood-brain barrier (BBB), Theiler's murine encephalomyelitis virus (TMEV), Peptide-induced fatal syndrome (PIFS), CD8 T cell, CNS vascular permeability

\section{Background}

Disruption of the blood brain barrier (BBB) is a common feature of several devastating immune-mediated neurological disorders, including multiple sclerosis, dengue hemorrhagic fever, cerebral malaria, stroke, and acute hemorrhagic leukoencephalitis (AHLE) [1-8]. The underlying molecular mechanisms of BBB disruption observed in these conditions remain poorly understood, undermining

\footnotetext{
* Correspondence: Johnson.Aaron2@mayo.edu

'Department of Neurology, Mayo Clinic, Rochester, MN, USA

2Department of Immunology, Mayo Clinic, Rochester, MN, USA

Full list of author information is available at the end of the article
}

the development of potential therapeutic approaches for human diseases characterized by BBB disruption. Our lab developed an inducible murine model of CNS vascular permeability using a variation of the Theiler's murine encephalomyelitis virus (TMEV) model commonly used to study multiple sclerosis. In this model, peptide-specific stimulation of CNS-infiltrating CD8 T cells in vivo initiates disruption of the $\mathrm{BBB}$, resulting in astrocyte activation, severe CNS vascular permeability, and functional motor deficits, followed by death within 48 hours. This peptide induced fatal syndrome (PIFS) is dependent on 
perforin expression, but does not rely on neutrophils, CD4 T cells, TNF- $\alpha$, IFN- $\gamma$, LT $\beta R$ or IL-1 $[9,10]$.

In addition to CNS-infiltrating CD8 T cells and their effector functions, there is potential for other genetic factors to contribute to BBB disruption. C57BL/6J (B6) and 129S1/SvImJ (129S1) mice both express the H2-D ${ }^{\mathrm{b}}$ class I molecule and thus are resistant to chronic TMEV infection and demyelinating syndrome [11-13]. However, despite having identical MHC class I molecules, epitopespecific CNS-infiltrating CD8 T cells, and equivalent cytotoxic T lymphocyte (CTL) perforin-mediated killing, B6 mice are highly susceptible to PIFS, whereas 129S1 mice remain resistant. This difference in susceptibility was illustrated by higher glial fibrillary acidic protein (GFAP) expression, increased CNS vascular permeability, and severe functional motor deficit in VP2 $121-130$-treated B6 mice when compared to VP2 $121-130$-treated $129 \mathrm{~S} 1$ mice. Furthermore, this susceptibility is transferable with the bone marrow compartment. 129S1 mice reconstituted with $\mathrm{B} 6$ bone marrow exhibit severe CNS vascular permeability, microhemorrhage formation, and functional deficit when compared to 129S1 mice receiving autologous bone marrow transfer [14]. Through an initial microsatellite analysis, we have determined that the PIFS phenotype is not mediated through a single gene, but rather is likely to be a complex condition [15].

In order to provide a more comprehensive analysis and determine the location of genes that influence the PIFS phenotypes, we analyzed the F2 generation of brother-sister mated F1 animals from a B6 and 129S1 cross using QTL analysis. The quantifiable traits of astrocyte activation via GFAP expression, CNS vascular permeability via fluorescent molecule diffusion across the $\mathrm{BBB}$, and functional motor deficits measured with rotarod activity were chosen for study because they differ by or exceed two standards of deviation when measured in the two parental strains, which is optimal for QTL analysis $[14,16]$. We identified chromosomal regions that harbored QTLs responsible for functional deficit and CNS vascular permeability. Additionally, we have identified candidate genes implicated in regulating these traits. These genes can be further investigated and may become potential targets for therapeutic approaches to ameliorate neurological disorders characterized by BBB disruption.

\section{Results}

As shown in Figure 1A, B6 mice were crossed with 129S1 mice to yield an F1 generation, and then F1 hybrid mice were brother-sister mated to produce 303 F2 progeny. These mice were intracranially infected with TMEV and then intravenously administered VP2 $2_{121-130}$ peptide 7 days later to induce PIFS. Mice were then analyzed for CNS vascular permeability, astrocyte expression of GFAP, and functional motor deficit (see Methods section). B6 mice were susceptible to each of these phenotypes, while 129S1 mice remained resistant [14]. F2 mice displayed variable susceptibility to each of these traits and were divided into susceptible and resistant groups based on whether they exhibited the trait or not. CNS vascular permeability was evaluated using a fluorescent plate reader to detect FITC-albumin leakage in brain homogenates using established methods $[14,17,18]$. Samples were read at $\mathrm{A}_{488}$, and a threshold value of 500 was used to determine susceptibility or resistance. Astrocyte expression of GFAP was detected through Western blot analysis as previously published $[14,18]$. Expression values were based on GFAP protein levels normalized to GAPDH. We used a threshold value of 50 to categorize F2 mice into susceptible and resistant groups. Functional motor deficit was assessed using the rotarod behavioral assay. A threshold value of $50 \%$ initial motor ability was used to categorize mice as susceptible or resistant (Additional file 1). The phenotype data for each trait is illustrated by the mean \pm SEM for the F2 susceptible group and the F2 resistant group (Figure 1B). No significant sex differences were observed in the F2 mice (data not shown).

\section{Identification of QTL controlling quantitative traits associated with PIFS}

Of the 671 SNPs analyzed, the most significant linkage to susceptibility to PIFS was observed with markers on chromosomes 12 and 17, as illustrated by log-likelihood plots (Figure 2). Significant LOD scores were obtained for functional motor deficits, which mapped to chromosome $12(\mathrm{LOD}=3.3)$ (Figure $2 \mathrm{~A})$, and $\mathrm{CNS}$ vascular permeability, which mapped to chromosome $17(\mathrm{LOD}=3.7)$ (Figure 2B). No significant LOD score was obtained for astrocyte activation as measured by GFAP expression (Figure 2C). Parental allele distribution of the linked SNPs within the QTL intervals that mapped to the phenotypes of functional motor deficits and CNS vascular permeability were detected using $2 \times 3$ contingency tables comparing B6 homozygous (BB), heterozygous (BS), and 129S1 homozygous (SS) mice. The QTL location on chromosome 12 (PIFS1, LOD 3.3), estimated by a $95 \%$ confidence interval, was from $4.7 \mathrm{cM}$ to $10.1 \mathrm{cM}$, with the peak of linkage at $6.0 \mathrm{cM}$. The QTL location on chromosome 17 (PIFS2, LOD 3.7), also estimated by a $95 \%$ confidence interval, was from $5.7 \mathrm{cM}$ to $12.5 \mathrm{cM}$, with the peak of linkage at $10.8 \mathrm{cM}$. We found that the 129S1 strain contributed to low rotarod performance, while the B6 strain contributed to high rotarod performance on these markers (Table 1A). In addition, the 129S1 strain contributed to increased CNS vascular permeability, whereas the B6 strain contributed to reduced CNS vascular permeability in the PIFS model (Table 1B). 


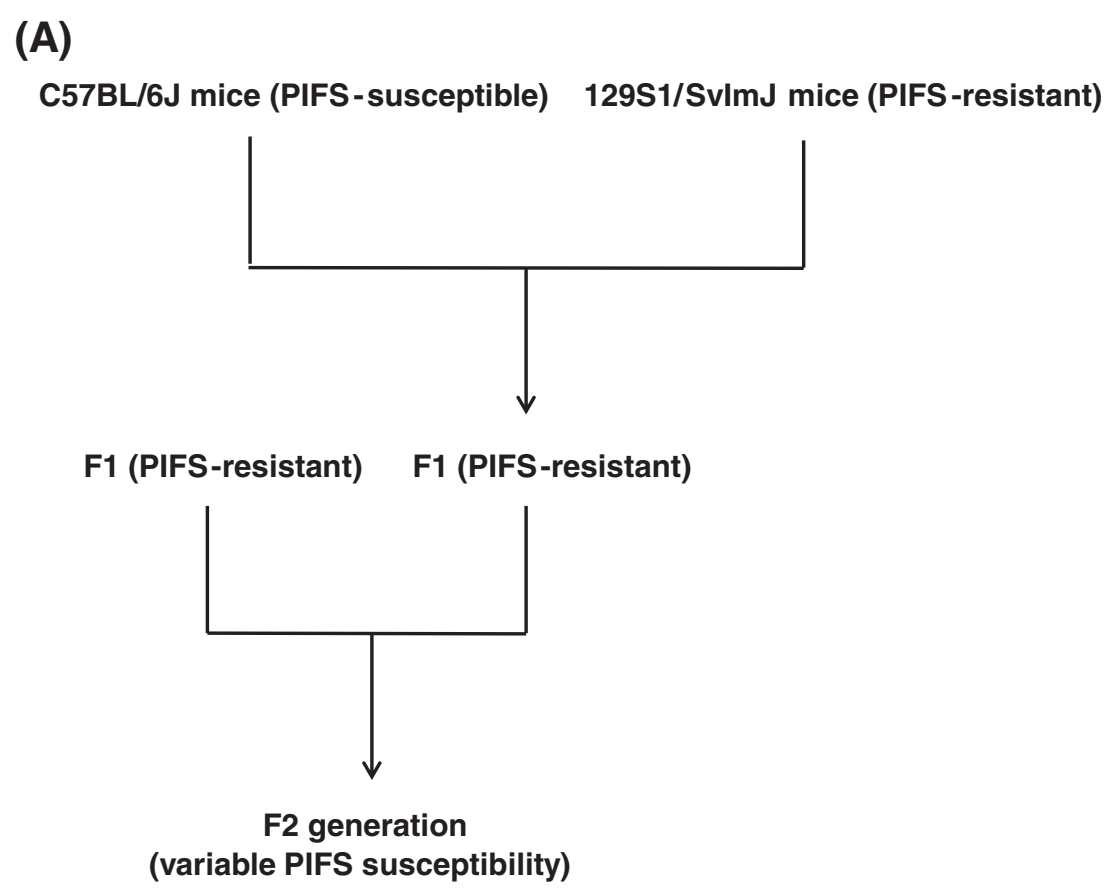
Analysis of: 1.) CNS vascular permeability
2.) Astrocyte expression of GFAP
3.) Functional motor deficit

(B)

\begin{tabular}{|c|c|c|}
\hline Phenotype & F2 Susceptible & F2 Resistant \\
\hline $\begin{array}{c}\text { CNS vascular permeability } \\
\text { (detection of FITC -albumin } \\
\text { leakage in brain homogenates) }\end{array}$ & $\begin{array}{c}737.3 \pm 23.2 \\
\left(\mathrm{~A}_{488}\right)\end{array}$ & $\begin{array}{c}251.7 \pm 10.4 \\
\left(\mathrm{~A}_{488}\right)\end{array}$ \\
\hline $\begin{array}{c}\text { GFAP expression } \\
\text { (Western blot analysis) }\end{array}$ & $\begin{array}{c}89.5 \pm 3.2 \\
\text { (GFAP:GAPDH ratio) }\end{array}$ & $\begin{array}{c}22.3 \pm 1.4 \\
(\text { GFAP:GAPDH ratio) }\end{array}$ \\
\hline $\begin{array}{c}\text { Functional motor deficit } \\
\text { (rotarod analysis) }\end{array}$ & $\begin{array}{c}8.3 \% \pm 1.2 \% \\
(\% \text { initial ability) }\end{array}$ & $\begin{array}{c}157.5 \% \pm 18.3 \% \\
(\% \text { initial ability) }\end{array}$ \\
\hline
\end{tabular}

Figure 1 Approach to map QTL for functional motor deficits, CNS vascular permeability, and astrocyte activation. (A) PIFS-susceptible B6 mice were crossed with PIFS-resistant 12951 mice to yield an F1 generation which was previously determined to be resistant to PIFS. F1 hybrid mice were then brother-sister mated to produce 303 F2 progeny with variable susceptibility to traits associated with PIFS, including functional motor deficits, astrocyte GFAP expression, and CNS vascular permeability measured by FITC-albumin leakage. DNA from 273 randomly selected F2 progeny were analyzed using Illumina Chip Technologies. SNPs with a known cM value were analyzed for the relationship between quantifiable traits (functional motor deficits, CNS vascular permeability, and astrocyte expression of GFAP) and genotype. (B) F2 mice displayed variable susceptibility to each of the traits associated with PIFS and were divided into susceptible and resistant groups based on whether they exhibited the trait or not. CNS vascular permeability was evaluated using a fluorescent plate reader to detect FITC-albumin leakage in brain homogenates. Samples were read at $A_{488}$, and a threshold value of 500 was used to determine susceptibility or resistance. Astrocyte expression of GFAP was detected through Western blot analysis. Expression values were based on GFAP protein levels normalized to GAPDH. We used a threshold value of 50 to categorize F2 mice into susceptible and resistant groups. Functional motor deficit was assessed using the rotarod behavioral assay. A threshold value of $50 \%$ initial motor ability was used to categorize mice as susceptible or resistant. All values are displayed as mean \pm SEM. 


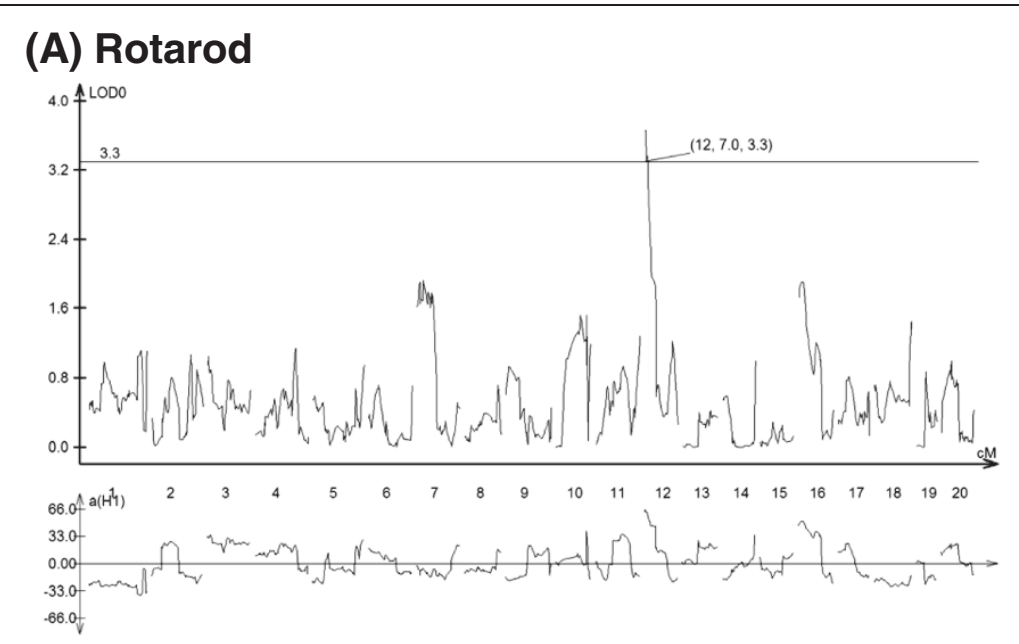

\section{(B) FITC Leakage}

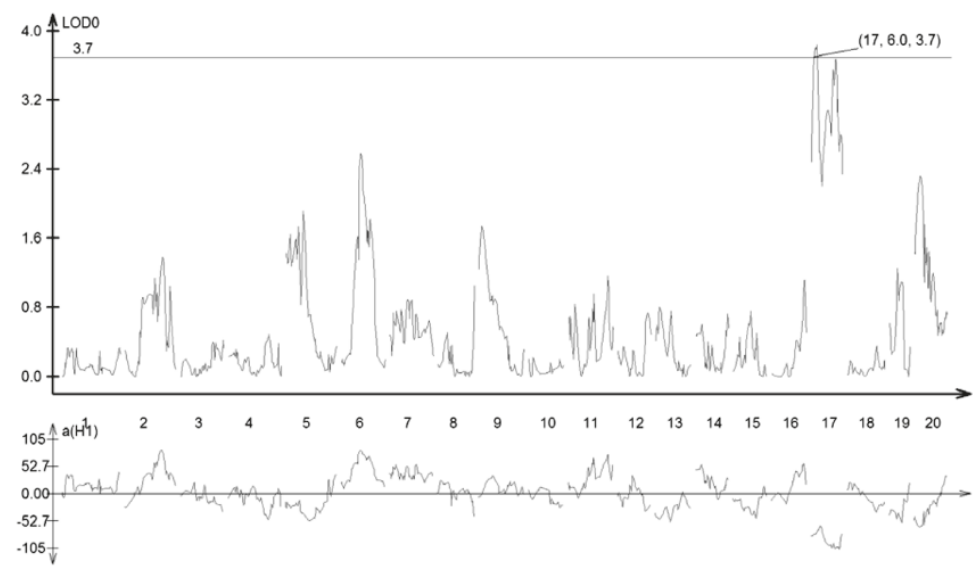

\section{(C) GFAP}

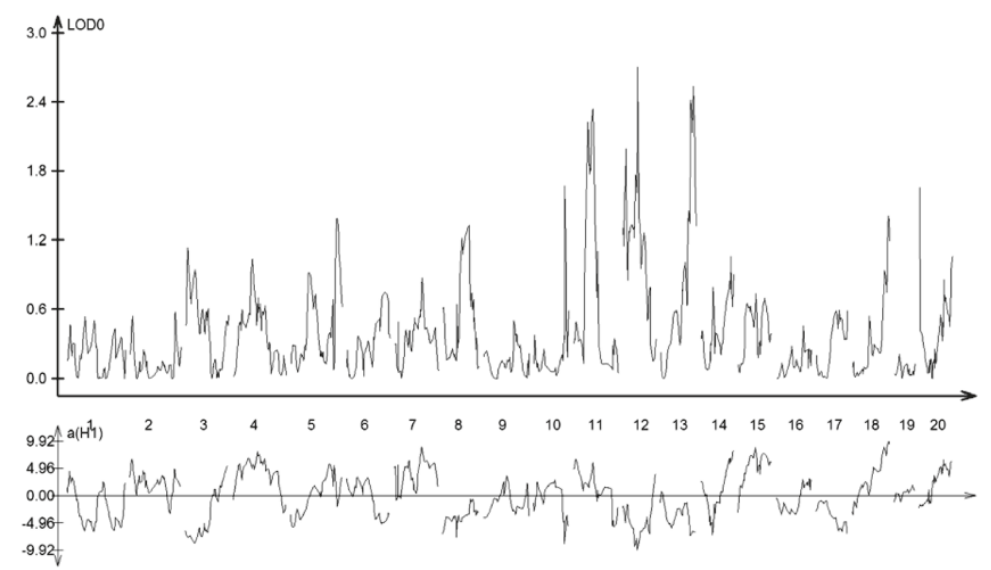

Figure 2 QTL analysis of functional motor deficits, CNS vascular permeability, and astrocyte activation. Log-likelihood plots of (A) functional motor deficits as measured by rotarod performance, (B) CNS vascular permeability as measured by leakage of FITC-albumin into the CNS, and (C) astrocyte activation as measured by GFAP expression. The upper graph charts the data as a LOD score. The solid black line depicts the significance threshold. The lower graph depicts the estimated QTL effects. Significant LOD scores were obtained for (A) functional motor deficits, which is shown to map to chromosome 12 ( $L O D=3.3$ ), and (B) CNS vascular permeability, which is shown to map to chromosome $17(\mathrm{LOD}=3.7)$. 
Table 1 Chi-square analysis of functional deficit and CNS vascular permeability

\begin{tabular}{|c|c|c|c|c|c|c|c|c|c|}
\hline \multirow[t]{2}{*}{ A. SNP } & \multicolumn{3}{|c|}{ Low F2s $(n=145)$} & \multicolumn{3}{|c|}{ High F2s $(n=128)$} & \multirow[t]{2}{*}{ Chi-square test } & \multirow[t]{2}{*}{ Chi-square $p$-value } & \multirow[t]{2}{*}{ LOD score } \\
\hline & BB & BS & SS & BB & BS & SS & & & \\
\hline rs6292954 & 18 & 80 & 47 & 50 & 51 & 27 & 25.93 & 0.000002 & 3.65 \\
\hline rs13481303 & 17 & 83 & 45 & 46 & 56 & 26 & 22.71 & 0.000012 & 3.66 \\
\hline rs3655057 & 19 & 80 & 46 & 46 & 54 & 28 & 19.66 & 0.000054 & 3.30 \\
\hline rs13481324 & 21 & 79 & 45 & 49 & 52 & 27 & 20.28 & 0.000039 & 3.35 \\
\hline \multirow[t]{2}{*}{ B. SNP } & \multicolumn{3}{|c|}{ Low F2s $(n=182)$} & \multicolumn{3}{|c|}{ High F2s $(n=91)$} & Chi-square test & Chi-square $p$-value & LOD Score \\
\hline & BB & BS & SS & BB & BS & SS & & & \\
\hline rs6196216 & 58 & 88 & 36 & 20 & 39 & 32 & 8.24 & 0.016245 & 3.73 \\
\hline rs3672065 & 60 & 85 & 37 & 19 & 39 & 33 & 9.27 & 0.009706 & 3.79 \\
\hline
\end{tabular}

$2 \times 3$ contingency tables comparing B6 homozygous (BB), heterozygous (BS), and 129S1 homozygous (SS) mice with regards to (A) functional motor deficits as measured by rotarod performance and (B) CNS vascular permeability as measured by leakage of FITC-albumin into the CNS. (A) Mice displaying <50\% initial ability were placed in the low F2 group, while mice displaying $>50 \%$ initial ability were placed in the high F2 group. 129S1 contributes to low rotarod performance on chromosome 12 markers rs6292954, rs13481303, rs3655057, and rs13481324, while B6 contributes to high rotarod performance on these markers. (B) The low F2 group consists of mice with FITC scores $<500$, while the high F2 group consists of mice with FITC scores $>500$. B6 contributes to less CNS vascular permeability on chromosome 17 markers rs6196216 and rs3672065, while 129S1 contributes to more CNS vascular permeability on these markers.

Potential candidate genes implicated in regulating functional deficit and CNS vascular permeability in CD8 T cell-initiated BBB disruption

Using the Mouse Genome Informatics Database resources (www.informatics.jax.org), we performed a search between the base pairs of the significant loci found on chromosome 12 (SNP markers rs6292954, rs13481303, rs3655057, and rs13481324) and chromosome 17 (SNP markers rs6196216 and rs3672065) in order to discover genes located in proximity with these loci. Our search revealed 34 candidate genes on chromosome 12 and 58 candidate genes on chromosome 17 (Table 2). We then prioritized these genes based on known or presumed biological function and previous studies, allowing us to narrow down this list to 6 genes of interest on chromosome 12 and 8 genes of interest on chromosome 17. We focused on genes exhibiting potential immunological, hematopoietic, apoptotic, or CNS-related functions since factors related to these processes are likely to play a role in CD8 T cell-initiated BBB disruption. We then used the Mouse Phenome Database (www.phenome.jax.org) to determine the number of SNPs in these genes of interest that are different between the B6 and 129S1 mouse strains as well as the location of these SNPs (Table 3).

\section{Discussion}

In this study, we investigated the role of genetic factors involved with CD8 $\mathrm{T}$ cell-initiated fatal BBB disruption. We previously demonstrated that despite having identical MHC class I molecules, epitope-specific CNSinfiltrating CD8 $\mathrm{T}$ cells, and similar levels of perforinmediated killing, the B6 strain is highly susceptible to PIFS, while the 129S1 strain remains resistant $[14,15]$. This susceptibility is transferable with the bone marrow compartment, as 129S1 mice reconstituted with B6 bone marrow exhibit characteristics of the PIFS phenotype, which include severe CNS vascular permeability, microhemorrhage formation, and functional motor deficit, when compared to $129 \mathrm{~S} 1$ mice receiving autologous bone marrow transfer [14]. Furthermore, by conducting a backcross of $(129 \mathrm{~S} 1 \times \mathrm{B} 6) \mathrm{F} 1$ mice, we determined through microsatellite analysis that susceptibility to PIFS is dictated in a complex fashion [15]. Therefore, we investigated the characteristics of PIFS in F2 generation mice through analysis of three quantifiable traits, which include functional motor deficit on rotarod, activation of astrocytes, and CNS vascular permeability. Since we have shown that the means of each of these traits measured between the B6 and 129S1 mouse strains differ by greater than two standards of deviation, we expected to find a set of QTLs for each trait measured [14].

We identified one novel QTL (PIFS1) on chromosome 12 linked to functional motor deficits and a second novel QTL (PIFS2) on chromosome 17 linked to CNS vascular permeability. While causality cannot be determined in this study, we have not observed motor dysfunction without CNS vascular permeability in the PIFS model. Furthermore, we discovered that the SNP markers identified within the QTL intervals for the PIFS-resistant 129S1 strain actually contributed to low rotarod performance and more CNS vascular permeability, while the B6 strain contributed to functional motor preservation and reduced CNS vascular permeability. While this was an unexpected result, it is possible that the expression of susceptibility loci may be suppressed by the presence of other loci in the parental strain. However, since new genetic combinations can be revealed through an F2 analysis, the identification of cryptic QTL becomes possible. For example, a genetic analysis of $(\mathrm{B} 6 \times \mathrm{FVB} / \mathrm{NJ}) \mathrm{F} 2$ progeny to study atherosclerosis revealed that the $\mathrm{FVB} / \mathrm{NJ}$ strain, which is more resistant to atherosclerosis than the B6 strain, actually contributed to high levels of the disease [19]. Therefore, 
Table 2 Candidate genes implicated in regulating functional deficit and CNS vascular permeability in fatal BBB disruption

\begin{tabular}{|c|c|c|c|c|c|c|c|}
\hline Gene & Chr & $\mathrm{cM}$ & Genome coordinates & Gene & $\mathrm{Chr}$ & $\mathrm{cM}$ & Genome Coordinates \\
\hline $\mathrm{Nt5c1b}$ & 12 & 5.27 & 10376779-10396979 (+) & Gm10513 & 17 & 7.8 & $11918744-11925211(-)$ \\
\hline Rdh14 & 12 & 5.29 & 10397586-10402368 (+) & Gm16168 & 17 & 7.8 & $10689312-10762262(+)$ \\
\hline 1700034J04Rik & 12 & 5.58 & $11228267-11229010(-)$ & Gm16169 & 17 & 7.8 & 11000700-11019491(+) \\
\hline 4930511A02Rik & 12 & 5.58 & 11445039-11451618 (+) & Pacrg & 17 & 7.8 & 10595878-11033177 (-) \\
\hline Gen1 & 12 & 5.58 & $11247732-11272593(-)$ & Park2 & 17 & 7.8 & $11033250-12256227(+)$ \\
\hline Kens3 & 12 & 5.58 & 11097008-11157648 (-) & Agpat4 & 17 & 8.33 & $12311570-12412511(+)$ \\
\hline Msgn1 & 12 & 5.58 & $11215188-11215754(-)$ & Map3k4 & 17 & 8.42 & 12420487-12511526 (-) \\
\hline Rad51ap2 & 12 & 5.58 & 11462885-11469734 (+) & 4732491K20Rik & 17 & 8.5 & $12511751-12520116(+)$ \\
\hline Smc6 & 12 & 5.58 & 11272692-11326591 (+) & $\mathrm{Plg}$ & 17 & 8.5 & $12571475-12612250(+)$ \\
\hline Vsnl1 & 12 & 5.58 & $11332053-11443455(-)$ & Slc22a3 & 17 & 8.52 & $12612838-12700570(-)$ \\
\hline Fam49a & 12 & 5.63 & $12268945-12383165(+)$ & C030013G03Rik & 17 & 8.56 & $12659710-12672444(+)$ \\
\hline Gm17337 & 12 & 6 & $12794791-12801091(+)$ & Slc22a2 & 17 & 8.61 & $12777055-12821331(+)$ \\
\hline 4930519A11Rik & 12 & 6.11 & 12909526-12914057 (+) & Slc22a1 & 17 & 8.63 & $12841740-12868704(-)$ \\
\hline Mycn & 12 & 6.14 & $12942902-12948720(-)$ & $\operatorname{lgf} 2 r$ & 17 & 8.64 & $12875272-12962530(-)$ \\
\hline Ddx1 & 12 & 6.4 & $13226113-13255980(-)$ & Airn & 17 & 8.66 & $12934177-13052988(+)$ \\
\hline Nbas & 12 & 6.45 & $13275933-13590616(+)$ & Gm19270 & 17 & 8.66 & $12926941-12946579(+)$ \\
\hline Fam84a & 12 & 6.93 & $14154405-14158844(-)$ & Gm17592 & 17 & 8.69 & 13030801-13031000 (-) \\
\hline Gm16497 & 12 & 7.03 & $14268340-14292490(+)$ & Mas1 & 17 & 8.69 & $13033945-13061009(-)$ \\
\hline Trib2 & 12 & 7.3 & $15798533-15823683(-)$ & Mrgprh & 17 & 8.7 & 13068900-13070708 (+) \\
\hline Gm5432 & 12 & 7.28 & 15823809-15894961 (+) & Pnldc1 & 17 & 8.71 & $13081768-13102866(-)$ \\
\hline Lpin1 & 12 & 7.9 & $16542475-16596576(-)$ & Acat3 & 17 & 8.72 & $13116699-13133268(-)$ \\
\hline Ntsr2 & 12 & 7.98 & 16660276-16667042 (+) & Mrpl18 & 17 & 8.72 & $13104215-13109211(-)$ \\
\hline Greb1 & 12 & 8 & $16677421-16807692(-)$ & Snora20 & 17 & 8.72 & $13115723-13115783(+)$ \\
\hline 2410004P03Rik & 12 & 8.04 & $17011764-17018360(-)$ & Tсp1 & 17 & 8.72 & $13108567-13117933(+)$ \\
\hline E2f6 & 12 & 8.04 & 16817771-16833549 (+) & Acat2 & 17 & 8.73 & $13135756-13153613(-)$ \\
\hline Kcnf1 & 12 & 8.04 & 17178906-17183694 (-) & Wtap & 17 & 8.73 & $13159662-13185412(-)$ \\
\hline Pqlc3 & 12 & 8.04 & 16995454-17007214 (-) & Sod2 & 17 & 8.75 & 13200705-13210985 (+) \\
\hline Rock2 & 12 & 8.04 & $16901784-16995080(+)$ & Gpr31b & 17 & 8.76 & 13244187-13245146(-) \\
\hline Atp6v1c2 & 12 & 8.06 & 17291527-17336165 (-) & Tcp10b & 17 & 8.76 & 13253970-13275347 (+) \\
\hline Pdia6 & 12 & 8.06 & $17273351-17291576(+)$ & Gm11166 & 17 & 8.78 & 13289526-13291338 (-) \\
\hline Nol10 & 12 & 8.07 & 17355299-17436901 (+) & Unc93a & 17 & 8.78 & $13301483-13324657(-)$ \\
\hline Mir3066 & 12 & 8.08 & $17362198-17362280(+)$ & Gm10512 & 17 & 8.81 & 13397908-13399077 (+) \\
\hline Gm19657 & 12 & 8.09 & 17436584-17441442 (-) & Smok2a & 17 & 8.82 & $13414054-13420524(+)$ \\
\hline Odc1 & 12 & 8.11 & 17551679-17558308 (+) & Smok2b & 17 & 8.82 & $13421718-13430055(+)$ \\
\hline Pde10a & 17 & 4.96 & 8718237-9179513 (+) & Tcp10c & 17 & 8.86 & 13547438-13570089(+) \\
\hline 1700010l14Rik & 17 & 5.53 & $9181198-9201184(+)$ & Rnu6 & 17 & 8.88 & 13624899-13625005 (-) \\
\hline 6530411M01Rik & 17 & 5.97 & 9340584-9360701 (-) & 2700054A10Rik & 17 & 8.9 & 13675419-13898832 (-) \\
\hline Gm17728 & 17 & 7.33 & $9614925-9615323(+)$ & Tcte2 & 17 & 8.9 & 13675419-13898832 (-) \\
\hline Gm17748 & 17 & 7.33 & 9614805-9622259 (-) & Gm16050 & 17 & 8.91 & 13695065-13704059 (+) \\
\hline Pabpc6 & 17 & 7.47 & $9859362-9862569(-)$ & Gm8603 & 17 & 8.91 & $13709618-13711010(+)$ \\
\hline Qk & 17 & 7.75 & 10399336-10512226 (-) & Smok4a & 17 & 8.91 & $13714322-13721299(+)$ \\
\hline A230009B12Rik & 17 & 7.8 & $10649082-10685921(+)$ & 4930488N24Rik & 17 & 8.95 & $14238826-14243457(-)$ \\
\hline
\end{tabular}

Table 2 Candidate genes implicated in regulating functional deficit and CNS vascular permeability in fatal

BBB disruption (Continued)

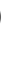


Table 2 Candidate genes implicated in regulating functional deficit and CNS vascular permeability in fatal BBB disruption (Continued)

\begin{tabular}{llll}
\hline Dact2 & 17 & 8.95 & $14332238-14340838(-)$ \\
Gm16046 & 17 & 8.95 & $13812667-13820523(-)$ \\
Gm16049 & 17 & 8.95 & $13896413-13897375(-)$ \\
Gm16052 & 17 & 8.95 & $13896377-13896992(-)$ \\
Gm7168 & 17 & 8.95 & $14085380-14087685(+)$ \\
Gm7356 & 17 & 8.95 & $14137246-14138709(-)$ \\
Gm7358 & 17 & 8.95 & $14195012-14196538(-)$ \\
Mllt4 & $\mathbf{1 7}$ & $\mathbf{8 . 9 5}$ & $\mathbf{1 3 8 9 7 5 4 6 - 1 4 0 4 3 1 5 7 ( + )}$ \\
\hline
\end{tabular}

Shown are a list of genes between the significant loci found on chromosome 12 (SNP markers rs6292954, rs13481303, rs3655057, and rs13481324) and chromosome 17 (SNP markers rs6196216 and rs3672065). Genes that exhibited potential immunological, hematopoietic, apoptotic, or CNS-related functions were categorized as genes of interest.

while our finding may seem surprising, it is important to note that disease-resistant strains also harbor susceptibility alleles, consistent with other genetic studies [19-21].

Identification of QTL regions that influence resistance and susceptibility to PIFS has enabled us to identify candidate genes that mediate protection from PIFS and thus are potential targets for therapy in diseases characterized by BBB disruption. Genes whose biological function had relevance to playing a role in $\mathrm{BBB}$ disruption were categorized as genes of interest and will be targeted first in future studies. Genes of interest pertaining to mouse functional ability on chromosome 12 include tribbles homolog 2 (Trib2), which has been shown to induce apoptosis of cells in hematopoietic origin. Additionally, mice homozygous for a null Trib2 allele display increased mean percentage of CD8 T cells. Furthermore, Trib2 has also been characterized as one of the top non-HLA markers associated with susceptibility to multiple sclerosis [22]. Considering our previous research on the critical roles of CD8 T cells and hematopoietic factors in mediating BBB disruption, Trib2 will be a candidate for future study $[9,10,14,15,17,18]$. Other genes of interest include Lpin1 and Rock2, which both play a role in organization of the actin cytoskeleton, $V s n l 1$, which plays a role in neuronal $\mathrm{Ca}^{2+}$ sensor proteins, Kcnf1, which plays a role in neurotransmitter release and neuronal excitability, and $D d x 1$, which plays a role in viral responses. Genes of interest on chromosome 17 that may affect CNS vascular permeability include Pde10a, Qk, Pacrg, Park2, Plg, Igf2r, Mas1, and Mllt4. These genes exhibit a variety of functions that may be involved in causing disruption of the BBB and ensuing CNS vascular permeability. For example, Mllt4 has been shown to play a role in adherens junctions and cell junctions. $\mathrm{Plg}$ has been demonstrated to be involved in apoptotic processes and vessel development. Additionally, Mas1 is involved in

Table 3 Genes of interest potentially related to B6 and 129S1 phenotypic differences in CD8 T cell-initiated BBB disruption

\begin{tabular}{|c|c|c|c|}
\hline Chromosome 12 (Rotarod) & Proposed function & $\begin{array}{l}\text { \# SNPs different between } \\
\text { strains }\end{array}$ & SNP locations \\
\hline Vsnl1 & Neuronal $\mathrm{Ca} 2+$ sensor proteins & 24 & All I \\
\hline $\mathrm{Dd} \times 1$ & Viral responses & 2 & All I \\
\hline Trib2 & $\begin{array}{l}\text { Induces apoptosis of cells in hematopoietic origin; } \\
\text { CD8 T cells }\end{array}$ & 40 & $1 \mathrm{Cs}, 1 \cup 5,38 \mathrm{I}$ \\
\hline Lpin1 & Demyelination; actin cytoskeleton reorganization & 531 & $\begin{array}{c}3 \mathrm{Cn}, 8 \mathrm{Cs}, 27 \mathrm{U3} \\
493 \text { । }\end{array}$ \\
\hline Kcnf1 & Neurotransmitter release; neuronal excitability & 35 & $4 \mathrm{Cs}, 7 \cup 5,24 \cup 3$ \\
\hline Rock2 & $\begin{array}{c}\text { Actin cytoskeleton organization; regulation of } \\
\text { angiogenesis }\end{array}$ & 330 & $\begin{array}{c}1 \mathrm{Cn}, 3 \mathrm{Cs}, 5 \mathrm{U} 3 \\
321 \mathrm{I}\end{array}$ \\
\hline $\begin{array}{c}\text { Chromosome } 17 \text { (CNS vascular } \\
\text { permeability) }\end{array}$ & Proposed function & $\begin{array}{l}\text { \# SNPs different between } \\
\text { strains }\end{array}$ & SNP locations \\
\hline Pde10a & Signal transduction; neuronal cell bodies & 1268 & Unspecified \\
\hline Qk & Myelinization; axon ensheathment; vasculogenesis & 214 & 12 U3, 202 I \\
\hline Pacrg & Myelinization & 265 & $2 \cup 5,2631$ \\
\hline Park2 & Neuron projection; neuron death & 3198 & 2 Cs, 1 U3, 3195 I \\
\hline Plg & Apoptotic processes; vessel development & 38 & $1 \mathrm{Cn}, 37 \mathrm{I}$ \\
\hline $\operatorname{lgf} 2 r$ & Edema & 7 & All I \\
\hline Mas1 & Inflammatory responses & 2 & All I \\
\hline Mllt4 & Adherens junctions; cell junctions & 7 & All I \\
\hline
\end{tabular}

Shown are the genes of interest found on chromosome 12 and chromosome 17 along with their proposed functions. The Mouse Phenome Database (www.phenome.jax.org) was used to determine the number of SNPs that are different between the B6 and $129 \mathrm{~S} 1$ mouse strains as well as their location. (Abbreviations: Cn nonsynonomous codon, Cs synonomous codon, U5 $5^{1}$ UTR variant, U3 $3^{1}$ UTR variant, I intron-variant). 
inflammatory responses, $\operatorname{Ig} 2 \mathrm{2} r$ is involved in edema, and $Q k$ and Pacrg both play a role in myelinization. Furthermore, Park2 has been shown to contribute to neuron projection as well as neuron death, and Pde10a has been shown to be involved in signal transduction and neuronal cell bodies (www.informatics.jax.org/). Furthermore, we used the Online Mendelian Inheritance in Man (OMIM) database (http://omim.org/) to investigate whether any of these genes of interest have been associated with any human neurological diseases. We found that Vsnl1, which is located on mouse chromosome 12, has been implicated in Alzheimer's disease. $Q k$, located on mouse chromosome 17, has been proposed to play a role in myelin and oligodendrocyte dysfunction in schizophrenia. Additionally, Pacrg, also located on mouse chromosome 17, may be involved in acquired, communicating hydrocephalus (http://omim.org/).

Our comprehensive genetic analysis has allowed us to create this list of candidate genes, which will serve as a critical starting point in elucidating the underlying molecular mechanisms of BBB disruption in neurological diseases. This will be an essential first step in developing potential therapeutic approaches to treat these diseases. Validating strain differences in primary DNA sequences in candidate genes and performing bioassays to test for functional consequences of primary DNA differences will be essential in this process. It will also be essential to test candidate genes for their ability to contribute to susceptibility or resistance to functional deficit and BBB disruption in the PIFS model. Testing specific congenic strains in which potential genes of interest will be introduced will help define the importance of the QTLs detected in this study. This approach has been successful in other genetic studies [23,24]. Generation of bacterial artificial chromosome (BAC) transgenic mice using the candidate genes we have identified will also be beneficial in evaluating the importance of our novel QTLs. This will enable us to test whether strain-specific variation in one or more of these genes is responsible for causing the QTL effects we observed [25]. This method has been carried out successfully in other genetic studies of strain comparison, such as evaluating the difference in seizure susceptibility between B6 and DBA/2 mouse strains [25]. Ultimately, results obtained from these experiments may hold promise for therapeutic intervention of immunemediated neurological diseases characterized by extensive BBB disruption.

\section{Conclusions}

The comprehensive genetic analysis performed in this study has enabled us to identify QTL linked to functional motor deficits and CNS vascular permeability, which are both devastating characteristics associated with CD8 T cell-initiated BBB disruption. Furthermore, identification of these critical QTL intervals has revealed a list of candidate genes implicated in regulating these traits. These findings are important because they will set the stage for future research to identify novel factors encoded by these genes that affect immune-mediated BBB disruption. This may ultimately hold promise for devising potential therapeutic approaches to treat the numerous immune-mediated neurological disorders characterized by $\mathrm{BBB}$ disruption.

\section{Methods \\ Animals}

Male and female C57BL/6J (B6) (stock\# 000664) and 129S1/SvImJ (129S1) (stock\# 002448) mice were obtained from Jackson laboratories (Bar Harbor, ME). $(\mathrm{B} 6 \times 129 \mathrm{~S} 1) \mathrm{F} 1$ hybrids were brother-sister mated to produce $(\mathrm{B} 6 \times 129 \mathrm{~S} 1) \times(\mathrm{B} 6 \times 129 \mathrm{~S} 1) \mathrm{F} 2$ progeny. All $\mathrm{F} 1$ and F2 mice were produced in the animal colony at the University of Cincinnati (Cincinnati, OH). In order to obtain large cohorts, mice were tested at 4-7 months of age. All experiments were approved by the Institutional Animal Care and Use Committee of the University of Cincinnati and Mayo Clinic.

\section{Induction of CNS vascular permeability using the PIFS model}

CNS vascular permeability was induced as previously described $[15,17,18]$. Briefly, all mice were intracranially infected with $2 \times 10^{6}$ PFU Daniel's strain of TMEV. 7 days post-TMEV infection, during the peak of CD8 T cell expansion, mice were intravenously administered $0.1 \mathrm{mg}$ VP2 121-130 (FHAGSLLVFM) peptide [15].

\section{Rotarod}

In order to evaluate functional motor deficits, mice were placed on the Rotamex-5 rotarod apparatus (Columbus Instruments) increasing from 5-40 RPMs over 7 minutes. Mice were trained twice daily for 3 consecutive days. The motor performance of mice was then assessed the day following this training period in order to establish baseline ability, which was considered the average of two trials. Mice were then injected with VP2 $121-130$ peptide to induce CNS vascular permeability and their motor performance was assessed again 24 hours later. Final scores were depicted as percent initial motor ability (day 2 average/day 1 average $\times 100$ ).

Fluorescein isothiocyanate (FITC)-albumin permeability assay Mice were given an intravenous injection of $10 \mathrm{mg}$ FITC-albumin (Sigma \#A9771) 23 hours post-VP2 121-130 peptide administration to induce PIFS. Whole brains were harvested one hour after administration of FITCalbumin and frozen on dry ice. Brain tissue samples were homogenized in radioimmunoprecipitation assay (RIPA) 
buffer $[10 \mathrm{mmol} / \mathrm{L}$ Tris, $140 \mathrm{mmol} / \mathrm{L} \mathrm{NaCl}, 1 \%$ Triton $\mathrm{X}-100,1 \% \mathrm{Na}$ deoxycholate, $0.1 \%$ SDS and protease inhibitor cocktail (Pierce \#78410) $\mathrm{pH}$ 7.5] and spun for $15 \mathrm{~min}$ at 10,000 RPM. Protein concentration was assessed using the BCA protein assay (Pierce \#23223). Samples were then normalized for protein content and read on a fluorescent plate reader at $488 \mathrm{~nm}$ excitation and $525 \mathrm{~nm}$ emission to detect FITC-albumin leakage into the brain. Data was collected using SpectraMax software (Molecular Devices).

\section{Western blot for glial fibrillary acidic protein (GFAP) detection}

Normalized protein samples from the FITC-albumin permeability assay were also used for western blot analysis. In order to detect GFAP, 10 ug of protein was loaded per well on 4-20\% precise protein gels (Pierce \#25244) with BupH Tris-HEPES-SDS running buffer (Pierce \#28398). Tris transfer buffer $[400 \mathrm{mmol} / \mathrm{mL}$ Tris base, $70 \mathrm{mmol} / \mathrm{mL}$ glycine, $10 \%$ methanol] was used to transfer gels onto Immun-Blot PVDF membranes (Biorad \#162-0177). GFAP was detected by staining with mouse anti-GFAP (1:1000 BD Pharmingen \#556329) and goat anti-mouse IgG conjugated to horseradish peroxidase (Sigma \#A3682). Scion Image Software (Scion Corp) was used to analyze Western blot films. Values were based on GFAP protein levels normalized to GAPDH $[14,18]$.

\section{Quantitative trait linkage analysis}

DNA from 273 F2 progeny (124 males; 149 females) were analyzed on 2 SNP chips using Illumina Chip Technologies. Two B6 and 2 129S1 mice were included as controls. In order to map genes controlling susceptibility to PIFS in the B6 $\times 129 \mathrm{~S} 1$ F2 model, we constructed a genetic linkage map of the mouse genome by analyzing 273 randomly selected mice using Illumina Chip Technologies. Of the 1449 analyzable SNPs, 874 were found to be unique between the two strains. 671 of these SNPs had a known cM value and thus were analyzed for the relationship between quantifiable traits and genotype [26]. Based on previous studies, we used functional motor deficits measured by rotarod, CNS vascular permeability measured by FITC-albumin leakage, and astrocyte activation measured by GFAP expression as quantifiable traits predictive of susceptibility to PIFS $[14,15]$. Threshold values were generated using the WinQTL Cartographer with 1,000 permutations of our data set to correct for multiple testing [27]. Interval mapping was used to identify significant linkage using a critical value of $\mathrm{p}=0.05$ and a $95 \%$ confidence interval (Additional file 2). A chi-square test statistic for each SNP marker showing linkage within a QTL interval was obtained using $2 \times 3$ contingency tables to demonstrate the distribution of parental strain alleles within the F2 population using the quantitative traits of functional motor deficits, CNS vascular permeability, and astrocyte activation. Since F2 mice displayed variable susceptibility, they were divided into two groups, low F2s and high F2s, based on whether or not they exhibited functional motor deficits or CNS vascular permeability. For functional motor deficits, low F2s were defined as those mice displaying $<50 \%$ initial ability, while high F2s were defined as mice displaying $>50 \%$ initial ability. For CNS vascular permeability, mice with FITC scores $<500$ were placed in the low F2 group while mice with FITC scores $>500$ were placed in the high F2 group for analysis. Since data were scored in a binomial fashion, we used a chisquare test, which is a nonparametric approach, to determine significance. We identified the critical interval for significant QTL and performed a search using www. informatics.jax.org to compile a list of candidate genes. These genes were then prioritized based on their known or presumed biological function. We then used the Mouse Phenome Database (www.phenome.jax.org) to determine the number of SNPs in these genes of interest that are different between the B6 and 129S1 mouse strains as well as the location of these SNPs. These genes will set the stage for future experiments on gene expression and strain differences that may be related to the mechanism of CD8 T cell-initiated BBB disruption.

\section{Availability of supporting data}

The data sets supporting the results of this article are included as additional files.

\section{Additional files}

\begin{abstract}
Additional file 1: Shown are the raw data obtained for CNS vascular permeability, as measured by assaying FITC-albumin leakage in brain homogenates, astrocyte expression of GFAP, as detected by Western blot analysis, and functional motor deficit, as assessed using the rotarod behavioral assay. Mice were divided into susceptible and resistant groups using the criteria stated in the text. Raw data values were used to determine mean \pm SEM for each trait.

Additional file 2: Shown are the raw data used to analyze the SNPs with known cM values for the relationship between quantifiable traits and genotype using WinQTL Cartographer. Mouse identification numbers are shown across the top. SNP markers, chromosome numbers, $\mathrm{CM}$ values, and genotype data are included. For genotype, $0=$ homozygous for B6, 1 = heterozygous, and 2 = homozygous for 12951 . Raw data for each quantifiable trait are included in a separate tab.
\end{abstract}

\section{Abbreviations}

BBB: Blood-brain barrier; TMEV: Theiler's murine encephalomyelitis virus; PIFS: Peptide-induced fatal syndrome; QTL: Quantitative trait loci; SNP: Single nucleotide polymorphism; FITC: Fluorescein isothiocyanate; GFAP: Glial fibrillary acidic protein.

Competing interests

The authors declare that they have no competing interests. 


\section{Authors' contributions}

HLJ performed rotarod behavioral assays, assisted with acquiring DNA and analyzing data, constructed the figures, and prepared the manuscript. LMH prepared DNA to be analyzed on the SNP chips, analyzed data using WinQTL Cartographer, and assisted with construction of figures. YC performed rotarod behavioral assays, assisted with acquiring DNA, and carried out western blots and FITC-albumin permeability assays. AJB assisted with analysis and interpretation of data. RJB assisted with analysis and interpretation of data and manuscript preparation. TNF contributed to revising the manuscript. IP participated in experimental designs and revising the manuscript. AJJ performed intracranial and intravenous injections, participated in acquiring DNA, and was involved in experimental design and preparing the manuscript. All authors read and approved the final manuscript.

\section{Acknowledgements}

We would like to thank the Genotyping Shared Resource of the Advanced Genomic Technology Center (Mayo Clinic, Rochester, MN) for assistance analyzing chips using Illumina Chip Technologies. We would also like to thank Kristina P. Bielewicz of the University of Cincinnati (Cincinnati, OH) for technical assistance. This work is supported by the National Institutes of Health grant NS060881 and Mayo Graduate School.

\section{Author details}

'Department of Neurology, Mayo Clinic, Rochester, MN, USA. ${ }^{2}$ Department of Immunology, Mayo Clinic, Rochester, MN, USA. ${ }^{3}$ Neurobiology of Disease Graduate Program, Mayo Graduate School, Rochester, MN, USA. ${ }^{4}$ Department of Neurology, University of Cincinnati, Cincinnati, OH, USA. ${ }^{5}$ Department of Neuroscience, Cooper Medical School of Rowan University, Camden, NJ, USA. ${ }^{6}$ Department of Psychiatry, University of Pennsylvania, Philadelphia, PA, USA.

Received: 11 March 2013 Accepted: 30 September 2013 Published: 3 October 2013

\section{References}

1. Brown H, Hien TT, Day N, Mai NT, Chuong LV, Chau TT, Loc PP, Phu NH, Bethell D, Farrar J, et al: Evidence of blood-brain barrier dysfunction in human cerebral malaria. Neuropathol Appl Neurobiol 1999, 25:331-340.

2. Huber JD, Egleton RD, Davis TP: Molecular physiology and pathophysiology of tight junctions in the blood-brain barrier. Trends Parasitol 2001, 24:719-725.

3. Lacerda-Queiroz N, Rodrigues DH, Vilela MC, Rachid MA, Soriani FM, Sousa LP, Campos RDL, Quesniaux VFJ, Teixeira MM, Teixeira AL: Platelet-activating factor receptor is essential for the development of experimental cerebral malaria. Am J Pathol 2012, 180:246-255.

4. Medana IM, Turner GD: Human cerebral malaria and the blood-brain barrier. Int J Parasitol 2006, 36:555-568.

5. Minagar A, Alexander JS: Blood-brain barrier disruption in multiple sclerosis. Mult Scler 2003, 9:540-549.

6. Nacer A, Movila A, Baer K, Mikolajczak SA, Kappe SH, Frevert U: Neuroimmunological blood brain barrier opening in experimental cerebral malaria. PLoS Pathog 2012, 8:e1002982.

7. Pirko I, Suidan GL, Rodriguez M, Johnson AJ: Acute hemorrhagic demyelination in a murine model of multiple sclerosis. J Neuroinflammation 2008, 5:31.

8. Talavera D, Castillo AM, Dominguez MC, Gutierrez AE, Meza I: IL8 release, tight junction and cytoskeleton dynamic reorganization conducive to permeability increase are induced by dengue virus infection of microvascular endothelial monolayers. J Gen Virol 2004, 85:1801-1813.

9. Johnson AJ, Njenga MK, Hansen MJ, Kuhns ST, Chen L, Rodriguez M, Pease LR Prevalent class I-restricted T-cell response to the Theiler's virus epitope $\mathrm{Db}$ : VP2121-130 in the absence of endogenous CD4 help, tumor necrosis factor alpha, gamma interferon, perforin, or costimulation through CD28. J Virol 1999, 73:3702-3708.

10. Johnson HL, Chen Y, Jin F, Hanson LM, Gamez JD, Pirko I, Johnson AJ: CD8 T cell-initiated blood-brain barrier disruption is independent of neutrophil support. J Immunol 2012, 189:1937-1945.

11. Njenga MK, Asakura K, Hunter SF, Wettstein P, Pease LR, Rodriguez M: The immune system preferentially clears Theiler's virus from the gray matter of the central nervous system. J Virol 1997, 71:8592-8601.
12. Rodriguez M, David CS: Demyelination induced by Theiler's virus: influence of the H-2 haplotype. J Immunol 1985, 135:2145-2148.

13. Rodriguez M, Pease LR, David CS: Immune-mediated injury of virusinfected oligodendrocytes - a model of multiple-sclerosis. Immunol Today 1986, 7:359-363.

14. Johnson HL, Chen Y, Suidan GL, McDole JR, Lohrey AK, Hanson LM, Jin F, Pirko I, Johnson AJ: A hematopoietic contribution to microhemorrhage formation during antiviral CD8 T cell-initiated blood-brain barrier disruption. J Neuroinflammation 2012, 9:60.

15. Johnson AJ, Mendez-Fernandez Y, Moyer AM, Sloma CR, Pirko I, Block MS, Rodriguez M, Pease LR: Antigen-specific CD8+ T cells mediate a peptide-induced fatal syndrome. J Immunol 2005, 174:6854-6862.

16. Jiang C, Zeng ZB: Mapping quantitative trait loci with dominant and missing markers in various crosses from two inbred lines. Genetica 1997, 101:47-58.

17. Suidan GL, Dickerson JW, Chen Y, McDole JR, Tripathi P, Pirko I, Seroogy KB, Johnson AJ: CD8 T cell-initiated vascular endothelial growth factor expression promotes central nervous system vascular permeability under neuroinflammatory conditions. J Immunol 2010, 184:1031-1040.

18. Suidan GL, McDole JR, Chen Y, Pirko I, Johnson AJ: Induction of blood brain barrier tight junction protein alterations by CD8 T cells. PloS one 2008, 3:e3037.

19. Moore KJ, Nagle DL: Complex trait analysis in the mouse: the strengths, the limitations and the promise yet to come. Annu Rev Genet 2000, 34:653-686.

20. Brodnicki TC, Quirk F, Morahan G: A susceptibility allele from a non-diabetes-prone mouse strain accelerates diabetes in NOD congenic mice. Diabetes 2003, 52:218-222.

21. Roper RJ, Weis JJ, McCracken BA, Green CB, Ma Y, Weber KS, Fairbairn D, Butterfield RJ, Potter MR, Zachary JF, et al: Genetic control of susceptibility to experimental Lyme arthritis is polygenic and exhibits consistent linkage to multiple loci on chromosome 5 in four independent mouse crosses. Genes Immun 2001, 2:388-397.

22. Baranzini SE, Wang J, Gibson RA, Galwey N, Naegelin Y, Barkhof F, Radue EW, Lindberg RL, Uitdehaag BM, Johnson MR, et al: Genome-wide association analysis of susceptibility and clinical phenotype in multiple sclerosis. Hum Mol Genet 2009, 18:767-778.

23. Ferraro TN, Golden GT, Smith GG, Martin JF, Schwebel CL, Doyle GA, Buono RJ, Berrettini WH: Confirmation of a major QTL influencing oral morphine intake in C57 and DBA mice using reciprocal congenic strains. Neuropsychopharmacology 2005, 30:742-746.

24. Ferraro TN, Smith GG, Schwebel CL, Lohoff FW, Furlong P, Berrettini WH, Buono RJ: Quantitative trait locus for seizure susceptibility on mouse chromosome 5 confirmed with reciprocal congenic strains. Physiol Genomics 2007, 31:458-462.

25. Ferraro TN, Golden GT, Dahl JP, Smith GG, Schwebel CL, MacDonald R, Lohoff FW, Berrettini WH, Buono RJ: Analysis of a quantitative trait locus for seizure susceptibility in mice using bacterial artificial chromosome-mediated gene transfer. Epilepsia 2007, 48:1667-1677.

26. Shifman S, Bell JT, Copley RR, Taylor MS, Williams RW, Mott R, Flint J: A high-resolution single nucleotide polymorphism genetic map of the mouse genome. PLOS Biol 2006, 4:e395.

27. Silva Lda C, Wang S, Zeng ZB: Composite interval mapping and multiple interval mapping: procedures and guidelines for using windows QTL cartographer. Methods Mol Biol 2012, 871:75-119.

doi:10.1186/1471-2164-14-678

Cite this article as: Johnson et al:: Quantitative trait loci analysis reveals candidate genes implicated in regulating functional deficit and CNS vascular permeability in CD8 T cell-initiated blood-brain barrier disruption. BMC Genomics 2013 14:678. 